\title{
Chromosomal Aberrations among Filipino Health Workers at the Chemotherapy Oncology Wards/ Clinics of a Tertiary Government Hospital
}

\author{
Corazon A. Ngelangel, ${ }^{1}$ Karen Villanueva-Timbol, ${ }^{1}$ Fatima dG. Fuerte, ${ }^{1}$ \\ Beatrice J. Tiangco, ${ }^{1}$ Susano B. Tanael Jr. ${ }^{1}$ and Ma. Luisa D. Enriquez ${ }^{2}$ \\ ${ }^{1}$ Section of Medical Oncology, Department of Medicine, College of Medicine and Philippine General Hospital, University of the Philippines Manila \\ ${ }^{2}$ Center for Natural Sciences and Environmental Research, De la Salle University, Taft Avenue, Manila
}

\begin{abstract}
Introduction. Chromosomal mutations are causal events in neoplasia development. Biomarker cytogenetic assays can determine exposure to mutagenic agents in occupational settings. This study assessed early biological marker chromosomal aberrations among health workers in the chemotherapy oncology wards/ clinics, exploring its association to the subjects' occupational, environmental and baseline profile.

Methods. This was an IRB approved cross-sectional exploratory study among hospital personnel working in the chemotherapy oncology facility of a tertiary government hospital, who underwent structured interview and blood extraction for cytogenetic assay after informed consent. Study funds only permitted assay of 44 specimens of 144 planned sample size, hence, Stata 6.0 only analyzed data from 44 subjects.
\end{abstract}

Results. All 44 subjects had varying exposure to chemotherapy drug infusions. Of these, $79 \%$ had $<0.8$ breaks per cell (hyposensitive), 7\% with 0.8-1.0 breaks per cell (borderline sensitive), and $14 \%>1.0$ breaks per cell (hypersensitive). Predominantly chromatid breaks (CTB), chromatid gaps (CTG), sister chromatid exchanges (SCE) were seen. No significant association was shown between mutagenic sensitivity and baseline characteristics, but with small sample size.

Conclusion. $21 \%$ borderline to hypersensitive mutagenic sensitivity among oncology workers at the tertiary government hospital is relatively significant, despite small sample size, connoting a must preventive promotive practice of chemotherapy administration in the workplace.

Key Words: chromosome aberrations, chemotherapy oncology clinics

Corresponding author: Corazon A. Ngelangel, MS, MD, PhD

Section of Medical Oncology

Department of Medicine

Philippine General Hospital

University of the Philippines Manila

Taft Avenue, Ermita, Manila 1000 Philippines

Telephone/Fax No.: +632 5263775

Email: corazon.ngelangel@gmail.com
Introduction

Molecular epidemiology in cancer is now gaining rapid growth and application in both basic and clinical research. Until recently the majority of cancer epidemiological studies have been limited to assessing possible causative associations between two types of events - exposure to potentially causative agents (i.e., cigarette smoke) and disease outcomes in terms of clinically apparent cancer incidence or cancer mortality. With the advancements in molecular biological research and technology, a multi-step process of carcinogenesis that lead to the development of this illness are becoming more and more understood. Cancer is now recognized as a genetic disorder and it is generally accepted that chromosomal mutations are causal events in the development of neoplasia.

Several types of biomarkers have now been developed that make it possible to monitor the progression of events from initial exposure (to a causative agent) to the development of a fully malignant tumor in a sequential fashion. Among these biomarkers are cytogenetic assays like chromosome aberrations, sister chromatid exchanges (SCE), micronuclei (MN), premature chromosome condensation and analysis of interphase cells for aneuploidy. These cytogenetic endpoints have been successfully used in epidemiologic studies for many years and they have been particularly useful in determining exposure to carcinogenic or mutagenic agents in occupational settings.

Chromosome aberrations (CA) are gross changes seen in chromosome structure as observed under light microscopy. SCE represent symmetrical exchanges between sister chromatids, and generally, they do not result in the alteration of chromosome morphology. MN represents small, additional nuclei formed by the exclusion of chromosome fragments and therefore indirectly reflect chromosome breakage. Recently performed epidemiologic studies show that CA frequency predicts the overall cancer risk in healthy subjects, ${ }^{1,2,3}$ while the association of SCEs and MN to carcinogenesis are still poorly understood. However, they are still important markers of pre-malignant cellular responses upon exposure to environmental mutagens and carcinogens. ${ }^{4}$ These very early responses have been found to occur at a higher rate in patients receiving chemotherapy 
and in doctors, nurses, and pharmacists occupationally exposed to such insults, compared to unexposed individuals whose chromosome abnormalities are considered as spontaneous aberrations.

Carcinogenic susceptibility has inter-individual differences that can be evaluated by biologic methods. Risk assessment is therefore recognized as a multidisciplinary process. Factors being considered in assessing cancer susceptibility are carcinogen metabolism and DNA repair capacity, which includes assessment of mutagen sensitivity. The latter is an in vitro assay that measures the frequency of mutagen-induced breaks in peripheral lymphocytes. There is a wide spectrum of DNA-repair capability within the population and thus a peripheral lymphocyte assay has been developed in which in vitro bleomycin-induced chromosome breaks provide an indirect measurement of such repair. Mutagen sensitivity as a determinant of susceptibility enables identify of high risk population subgroups as a determinant of susceptibility enables identity high risk population sub-groups that can be targeted for intensive primary and secondary preventive strategies.

Patients who have undergone chemotherapy treatment have an increased risk of developing chemotherapy related hematologic malignancies and solid tumors anywhere from one to ten years after receiving anti-neoplastic drugs. Studies establishing this fact are numerous and well designed. Early on, it was assumed that since post chemotherapy patients have a higher risk of developing secondary cancers compared to the general population, and genetic mutations are early biologic events in carcinogenesis, then a higher proportion of post-chemotherapy patients compared to the general population would have these mutations after chemotherapy. This has been proven in numerous studies.5,6

Because of the various studies published from the early 1980's proving the existence of chemotherapy related secondary malignancies, and the increase in markers of early biologic effects post chemotherapy, concerns arose about the hazards of occupational exposure to cytotoxic/ cytostatic chemotherapeutic agents. Several studies during the 1980s and 1990s showed increase in markers of biological effects after exposure to chemotherapy among health workers. These studies, many originating from Europe, commonly involved oncology nurses and, occasionally oncology pharmacists, as subjects. They generally had small sample size and the results generated were varied.

Jacobsen-Kram D et $\mathrm{al}^{7}$ measured various indicators of DNA damage in peripheral lymphocytes of human populations occupationally exposed to carcinogens (firefighters, cancer patients, undergoing chemotherapy and oncology pharmacists). They found increased levels of SCEs, HPRT mutations and DNA adduct formation in their study population but gave no data regarding their control groups. Cooke $\mathrm{J}$ et $\mathrm{al}^{8}$ published the result of a small pilot study which aimed to determine the baseline level of chromosomal damage produced by environmental exposure to cytotoxic agents in four groups of subjects: oncology pharmacy personnel, nurses in the oncology units, unexposed office workers and patients receiving cytotoxic drugs. Results showed a significantly greater damage in patients compared to the other three groups.

The preceding studies suggest possible carcinogenic and mutagenic risk for health professional that are occupationally exposed to these known carcinogens.

Because of these findings, strict guidelines regarding the use of protective equipment in the preparation and handling of chemotherapeutic agents have been implemented all over the world. These guidelines include preparation of chemotherapy agents only by trained personnel under specialized ventilated hoods and the use of gowns, gloves, and protective eyewear. These guidelines however are not being followed routinely in the Philippines. There is no public health policy regarding the preparation of cytotoxic materials in specialized areas and the use of protective equipment by all handlers of such drugs. The most that health workers utilize to protect themselves would be the use of gloves when preparing and administering chemotherapy agents. In informal group discussions and key informant interviews of health personnel in the Oncology Section of different tertiary hospitals in Metro Manila reasons for non-compliance with guidelines regarding use of protective equipment include lack of such protective equipment, unawareness of the possible hazards of occupational exposure and lack of time to seek and put on protective equipment. An unpublished study done by Tiangco and Tiambeng et al, involving health professionals administering chemotherapeutic agents in different oncologic units in Metro Manila, showed that 95\% of respondents were aware of the risks associated with the handling of cytotoxic chemotherapy but only 63\% actually use at least one protective equipment. Nurses and younger doctors were more likely to use protective equipment compared to older physicians.

To date, no local studies have been published to measure the presence of early biologic markers of carcinogenesis among health workers working in chemotherapy oncology facility.

This study explored the early biologic markers among Filipino health workers working in the chemotherapy oncology wards/ clinics in a tertiary government hospital, especially chromosomal aberrations in peripheral lymphocytes taken from the health workers.

\section{Methods}

This cross-sectional exploratory study included a preselected population who gave their informed consent and who were health workers at the oncology wards and outpatient clinics of the Philippine General Hospital, Manila. 
Excluded were those diagnosed with hematologic illness or cancers and disorders like multiple myeloma, leukemia, myelodysplasia, with febrile illness in the past three days, debilitating, chronic illness, documented chromosomal abnormalities, treated with chemotherapy 6 months prior to enrollment, and or treated with radiotherapy anytime in the past.

Sample size calculations were based on estimated mean mutation frequency (MF) of 4.6 for the zero exposed groups to 10.00 for the group with the highest exposure and within group standard deviation of 8.00. The test of equality of means will be carried out at the 0.05 level of significance. A sample size of 36 per group (x 4 groups [unexposed, mildly, moderate, highly exposed to cytotoxic chemotherapy drugs] $=144$ total) gave a probability of 0.086 of rejecting the null hypothesis of equal means if the alternative held. A total of 100 subjects were interviewed of whom 77 blood samples were taken, but only 44 blood samples were processed due to lack of funds; while waiting for approval of additional funding which failed, the rest of the blood samples become old after 2 years, unfit for laboratory evaluation.

The independent variables are duration of exposure to chemotherapy drugs, age, co-morbidities, diet, family/ personal history of cancer, exposure to carcinogens, smoking, alcohol; the dependent variable is the chromosomal aberrations per cell (chromosomal aberrations, SCE computed as total chromosomal breaks divided by 50 cells). Funds prevented the addition of $\mathrm{MN}$ as another biomarker.

From initial interview until disclosure of results, confidentiality was assured. During disclosure to positive subjects, they were offered additional counseling, should they wish to have this.

Assay of chromosome aberrations needed 5 cc of whole blood added to 45 cc of RPMI 1640 culture media with supplemental $10 \%$ fetal calf serum, L-glutamine $(2 \mathrm{mM})$, penicillin $(50 \mathrm{u} / \mathrm{ml})$ and streptomycin $(50 \mathrm{ug} / \mathrm{ml})$ and divided into sets of $10 \mathrm{cc} /$ flask. Phytohemaglutinin was added to each culture and maintained at $37^{\circ} \mathrm{C}$ in an atmosphere of $\mathrm{CO}^{2}$ in air. Cultures were harvested 48 hours later, and colcemid (10 uM) was added to each flask for the final 6 hours of culture. Hypotonic solution (0.1 M sucrose) was added for 3 minutes. After fixation in two changes of methanol:acetic acid (3:1), the cells were dropped into wet microslides and stained with Giemsa in Sorensen's buffer. Slides were analyzed under light microscope and 100 second division metaphases were cored for each subject. Assay of SCE needed the same protocol for blood extraction and cell plating but for the SCE group, 20\% 5-Brmodeoxyuridine (final concentration $20 \mathrm{uM}$ ) was added. After another replication cycle, coclemid was added and cultures were harvested. Differential staining of BrdUrd substituted chromosomes using $5 \mathrm{ug} / \mathrm{mL}$ Hoechst 33258 in $0.067 \mathrm{M}$ Sorensen's buffer ( $\mathrm{pH}$ 6.8) for 20 minutes was done. Then this was mounted in the same buffer with a coverslip and exposed to Blacklight $\left(23.8 \mathrm{~J} / \mathrm{m}^{2} / \mathrm{s}\right)$ on a $56^{\circ} \mathrm{C}$ hot plate for 30 minutes and then stained with 3\% Giemsa. One hundred second division metaphases were scored for SCE's per subject. Total CAs were sub-classified as CSAs (including chromosome-type breaks, ring chromosomes, marker chromosomes, double minutes (DM) and dicentrics (DIC)) and chromatid-type aberrations (CTAs; including chromatid-breaks and chromatid exchanges). Gaps were not scored as aberrations. Assays were done at the Biology Department of the De La Salle University, Taft Avenue, Manila.

This paper evaluated data from 44 subjects and did away with the 4 groups mentioned earlier. Descriptive statistics were computed for each quantitative independent variable while qualitative independent variables were described using percentages. Statistical data analysis was done using Stata 6.0 statistical software with independent variables age, sex, smoking history, diet, occupation, occupation practice, exposure to other carcinogens and comorbid conditions, against degree of mutagenic sensitivity (hyposensitive $(<0.8$ breaks per cell), borderline sensitive (0.8-1.0 breaks per cell), and hypersensitive ( $>1.0$ breaks per cell). Mutagenic sensitivity was reclassified into two categories namely - hyposensitive and sensitive (borderline + hypersensitive). Bivariate analysis using the outcome of interest CTG, CTB, SCE, CSB, DIC and DM for each category of mutagenic sensitivity was done. A one-way ANOVA was done to test for significant differences in mean CTG, CTB, SCE, CSB, DIC according to mutagenic sensitivity at a $5 \%$ level of significance.

\section{Results}

\section{Baseline Study Population Profile}

Forty-four patients were included in this study, with mean age of 34 years (19-66 years). Forty-eight percent were within age range 26-35 years. Of these, 27 (61\%) were females and 17 (39\%) were males. Nurses comprised the majority of the participants at $32 \%$, followed by postresidency fellows-in-training (25\%), nurse assistants $(23 \%)$, consultants $(11 \%)$, institutional worker $(5 \%)$, others $(4 \%)$. They had worked in the cancer ward or clinic for a mean duration of $50.8 \pm 68.8$ sd months, directly handling/ administering chemotherapy to about $10-11$ patients per week, with a duration mean of $2.5 \pm 4.2$ sd days giving chemotherapy to $\geq 3$ patients a week.

In terms of usage of protective gear in handling cytotoxics, 30\% always wore gloves while $27 \%$ used them more often than not. Thirty six percent wore masks sometimes, $14 \%$ always, and 25\% never did. Sixty-four percent had never worn a gown, with only $2 \%$ always.

Few subjects had past/ present history of diseases, namely - thyroid (5), hypertension (1), NIDDM (1) and iron deficiency anemia (1). 
Exposures to other environment risks factors for cancer cited frequently - smoked preserved food (61\%), cured food and polluted outside air (each 54\%), salted preserved food $(52 \%)$, pesticides and radioactive iodine $(23 \%)$,

Personal history showed majority (84\%) were lifelong non-smokers. The rest had smoked an average of 5.1 years duration. Forty-five percent were non-alcoholic beverage drinkers. All except one were meat eaters.

Forty-five percent had family history of cancer.

Pertinent cancer risk factors present were obesity (2), fibroadenoma (1), past history of cancer (1), helicobacter pylori infection (1), gastrointestinal surgery (1), RAI treated hyperthyroidism (1), and nodular goiter (1).

\section{Chromosomal Aberration \& Mutagenic Sensitivity}

All 44 subjects had chromosomal aberration assay showing - 79\% hyposensitive, $7 \%$ borderline, and $14 \%$ hypersensitive. All types of chromosomal aberration were seen among the subjects (Table 1), predominantly CTG, CTB, and SCE.

Table 1. Chromosomal Aberrations among Oncology Workers $(\mathrm{n}=44)$

\begin{tabular}{lrc}
\hline Biomarkers & Mean & SD \\
\hline CTB+SCE+CSB+DIC+DM & 27.64 & 21.56 \\
Chromatid-type breaks (CTB) & 16.52 & 13.88 \\
Sister Chromatid Exchanges (SCE) & 7.18 & 11.91 \\
Chromatid gaps (CTG) & 20.96 & 19.95 \\
Chromosome-type breaks (CSB) & 1.46 & 3.24 \\
Dicentrics (DIC) & 1.36 & 2.58 \\
Double minutes (DM) & 0.23 & 0.77 \\
\hline
\end{tabular}

The trend was for more CTG, CTB, SCE occurring among the hypersensitive group (Table 2).

Table 2. Chromosomal Aberrations by Mutagenic Sensitivity $(\mathrm{n}=44)$

\begin{tabular}{|c|c|c|c|c|}
\hline \multirow{2}{*}{$\begin{array}{l}\text { Chromosomal } \\
\text { Aberration }\end{array}$} & \multicolumn{3}{|c|}{ Mutagenic Sensitivity (mean+sd) } & \multirow[b]{2}{*}{ p-value } \\
\hline & $\begin{array}{c}\text { Hyposensitive } \\
\text { (35) }\end{array}$ & $\begin{array}{c}\text { Borderline } \\
\text { (3) }\end{array}$ & $\begin{array}{c}\text { Hypersensitive } \\
\text { (6) }\end{array}$ & \\
\hline CTG & $14.5 \pm 12.1$ & $47.7+9.2$ & $54.2 \pm 31.1$ & 0.000017 \\
\hline СТВ & $13.6 \pm 10.1$ & $26.7 \pm 15.5$ & $28.5 \pm 23.9$ & 0.0183 \\
\hline SCE & $2.9 \pm 4.0$ & $10.7 \pm 6.1$ & $30.7 \pm 17.3$ & $<0.0001$ \\
\hline CSB & $1.0 \pm 3.1$ & $2.0 \pm 3.5$ & $3.7 \pm 3.7$ & 0.18 \\
\hline DIC & $1.1 \pm 2.5$ & $4.0 \pm 4.0$ & $1.3 \pm 2.1$ & 0.19 \\
\hline $\mathrm{DM}$ & $0.2 \pm 0.8$ & $1.3 \pm 1.2$ & - & 0.03 \\
\hline
\end{tabular}

No significant association was shown between mutagenic sensitivity and study population characteristics, but with small sample size (Table 3).

Characteristics of the 6 hypersensitive subjects showed the following - mean age of 36 years, equal male-female ratio, varying occupation, spent an average of 45.8 months (range 6-120) in a cancer unit, 2 were smokers, 3 were alcoholic drinkers, 4 with family history of cancer. Four directly administered cytotoxics while 2 had indirect exposures. They had varying practices as to the use of gloves, masks and gowns. Most commonly cited environmental exposures were nitrates, smoked/salted food, $\mathrm{x}$-rays, paint, and polluted air.

Table 3. Subject Profile versus Mutagenic Sensitivity Simple Logistic Regression $(\mathrm{n}=44)$

\begin{tabular}{lc}
\hline \multicolumn{1}{c}{ Independent Variable } & P-value \\
\hline Age & 0.611 \\
Sex & 0.689 \\
Occupation & 0.471 \\
Duration work in oncology clinic/ ward & 0.760 \\
Duration give anti-cancer drugs to $\geq 3$ patients a week & 0.976 \\
Use gloves & 0.668 \\
Use mask & 0.636 \\
Use gown & 0.119 \\
Duration work in general wards & 0.756 \\
Duration in general OPD & 0.807 \\
With thyroid disease & 0.979 \\
Take high temperature soup/ liquid eating & 0.400 \\
Take nitrates/ nitrites (cured foods) & 0.169 \\
Take smoked preserved foods & 0.332 \\
Take salted preserved foods & 0.132 \\
Exposed to pesticide & 0.400 \\
Exposed to Radioactive iodine for treatment & 0.094 \\
Exposed to polluted air & 0.932 \\
Exposed to paint (painters) & 0.204 \\
Smoke cigarette/ tobacco & 0.976 \\
Drink alcohol & 0.417 \\
With family history of cancer & 0.163 \\
\hline
\end{tabular}

\section{Discussion}

The frequency of chromosomal aberrations in human peripheral blood lymphocytes measured with the conventional assay in metaphase cells has routinely been used for several decades as a tool to monitor occupational and environmental exposure to genotoxic carcinogens. ${ }^{9}$

This study explored the frequency of chromosomal aberrations among Filipino health workers at the oncology wards/ clinics. All subjects had some chromosomal aberrations (mean of 27.64+21.56 $s d$ ), predominantly CTBs (mean of 28.50+23.91 sd) and SCEs (mean of $30.67 \pm 17.28 \mathrm{sd}$ ). But, there were only 9 subjects with borderline (3) to hypersensitive (6) mutagenic sensitivity, with predominantly CTBs (mean of $16.52+13.88$ sd) and SCEs (mean of $7.18+11.91 \mathrm{sd}$ ); of these, $67 \%$ have a family history of cancer, $67 \%$ directly administered chemotherapy, 50\% were moderate alcoholic drinker, 33\% smokers, with mean age of 36 years and mean 45.8 months in a cancer unit.

Several studies had shown more aberrant cells in chemotherapy exposed groups. Medkova $\mathrm{J}^{10}$ did cytogenetic analysis of peripheral lymphocytes of doctors and nurses who handled cytostatic chemotherapy agents, finding a significantly higher number of aberrant cells compared to the control group $(\mathrm{p}<0.05)$. Norppa et $\mathrm{al}^{11}$ studied 15 nurses in daily contact with cytostatics and this group had higher mean number of SCEs compared to controls. However, the mean number of SCEs found among oncology nurses was not significantly different from that of other hospital nurses. 
A Brazilian study ${ }^{12}$ showed higher frequencies for CA and SCE rates in 15 nurses and nurses' aides working in an oncology unit compared to those working in other hospital units. A Hungarian study ${ }^{13}$ on clinical, geno- and immunotoxicology risk assessment of 500 nurses, showing higher frequencies of CA, SCE, and B lymphocytes compared to controls. There was also increase in prevalence of iron-deficiency anemia and thyroid gland disease among the subjects.

Medvoka $\mathrm{J}$ et $\mathrm{al}^{10}$ showed that nurses who have been exposed $>11$ years had a higher number of aberrant cells compared to the control group. However in this current study, occurrence of more chromosomal aberrations was not associated with duration of chemotherapy exposure even if the practice of protective gloves, mask, and gowns was lacking. Benhamou et $\mathrm{al}^{14}$ found that the individual mean number of SCE was significantly associated with the total number of drugs handled, and not necessarily the nature of these drugs or the duration of exposure. In contrast, Kasuba et al ${ }^{15}$ utilized $\mathrm{MN}$ and SCE in evaluating 20 oncology nurses and showed that those with longer exposure (20-31 years) to chemotherapy had higher MN frequency. Those with less exposure (1-14 years) had results comparable with the control. SCE was not significantly different among the different groups studied.

In this current study, there was a relatively short duration of exposure to chemotherapy administration (mean $2.5+4.2$ sd years) of different drug forms.

Jakab et $\mathrm{al}^{16}$ made a cytogenetic analysis of 4 groups of 95 nurses exposed to cytostatics, grouped mainly according to presence or absence of adequate biological safety cabinets (BSC) that are recommended for chemotherapy preparation. The first two groups (which had no or inadequate BSC, respectively) had significantly raised CA and SCE values. Those in the $3^{\text {rd }}$ group had lower levels compared to the former during initial investigations. However, during subsequent follow-ups, there were fluctuations in CA and high-frequency SCE in years 4 and 6 . In the $4^{\text {th }}$ group, these two markers were elevated despite use of BSC, suggesting other possible routes of exposure.

Perhaps due to the small size of the study population in this current study, there was no association shown between baseline characteristics (e.g., age, sex, presence of environmental/ medical risk factors) and degree of mutagenic sensitivity.

Fenech $\mathrm{M}^{17}$ showed that aging can explain at least $25 \%$ of variation in chromosomal damage rate in lymphocytes from both males and females. Bolognessi et al $^{18}$ confirmed age-related increase of baseline frequencies of said damage, with most dramatic increase seen in $\mathrm{MN}$, followed by $\mathrm{CA}$ and SCE.

There was no significant association seen in this current study for age and mutagenic sensitivity perhaps because the study population was young (mean $34.39 \pm 0.26$ sd years).
The current study population had relatively low exposure to environmental carcinogens and medical risk factors, probably hence no association was seen with degree of mutagenic sensitivity.

Rossner $\mathrm{P}$ et $\mathrm{al}^{9}$ in their study of 2,150 subjects exposed to cytostatic agents with cytogenetic data showed that the predictiveness of chromosomal aberrations observed in subjects exposed to various classes of carcinogens did not significantly differ from the group of non-exposed subjects. The Rossner study was in contrast with several studies: a) Lithuanian study ${ }^{19}$ involving 1,113 subjects evaluated cytogenetic effects of heavy metals, organic and inorganic substances (e.g., mercury, lead, styrene, benzopyrene, sulfur oxide, among others), comparing people with occupational or environmental exposure with controls, showing increased CA frequencies; b) air pollutions, if at moderate levels, did not significantly contribute to genetic damage ${ }^{20}$ while rubber exposure resulted to increased SCE frequency ${ }^{21}$. Smoking, when added to air pollution or rubber, added to chromosomal damage; c) cytogenetic damage had likewise been documented in exposure to plastics and paints; ${ }^{22,23} \mathrm{~d}$ ) Cardoso et $\mathrm{al}^{24}$ did cytogenetic analysis in peripheral blood lymphocytes from hospital workers chronically exposed to ionizing radiation, showing higher frequencies of chromosomal aberrations for the exposed group compared to control group; e) Donbak et $\mathrm{al}^{25}$ studied genotoxic risk of textile dryers, and noted higher CA frequency in the exposed group, with significant correlation with length of exposure; f) exposure to pesticides was noted to increase frequency of CA and MN in 27 Yugoslav vineyard growers compared to controls. ${ }^{26}$

In the current study, most of the subjects were non-smokers and only 2 of the 6 hypersensitive group members were smokers.

In a study of the general population in 3 Italian cities, mean values of SCE were directly related to number of cigarettes consumed per day, and in former smokers inversely related to length of time of smoking cessation. ${ }^{27}$ The effect of smoking in increasing frequency of SCE was also seen in a study by Sardas et al. ${ }^{28}$ In contrast, Benhamou et $\mathrm{al}^{15}$ on 29 oncology nurses showed no significant cytogenetic damage (based on SCE and CA) between the subjects and the controls per se. However, SCEs were more frequent in smokers (8.23) compared to non-smokers (6.75). They also found that the individual mean number of SCE was significantly associated with the total number of drugs handled, and not necessarily with the nature of these drugs or the duration of exposure.

In the current study, half of the subjects drunk alcohol however moderately. Alcohol is mutagenic, carcinogenic and teratogenic in man in more than moderate quantity. Acetaldehyde, the first metabolite of ethanol, induces CAs, SCEs, and cross-links between RNA strands. ${ }^{29}$

In summary, chromosomal aberrations were seen in all 44 subjects of this study, with 6 having hypersensitivity as measured by the biomarker, particularly CTBs and SCEs. Although only 
$14 \%$ of the health workers were found to have significant mutagenic sensitivity, there were another $7 \%$ with borderline sensitivity. Time, age, administration of chemotherapeutic drugs, family history of cancer might have played a role.

The finding of $21 \%$ borderline to hypersensitive mutagenic sensitivity among workers in the Philippine oncology workplace is relatively significant connoting a potential to develop a ratherhigh-mortality-profile disease, despite the small study sample size. Such a screen may be done to ascertain such risk. But screen or not screen, preventive promotive practice of anti-cancer chemotherapy administration in the workplace must always be the rule. All 44 subjects were informed of the results of this study and the importance of caution in the workplace. Not all subjects preferred to ask to which sensitivity they belong, knowing the $21 \%$ study result, but all have taken a note to be more extra careful. The Cancer Institute of the Philippine General Hospital has also been informed of the results of this study; lectures on safe chemotherapeutic handling and administration have been given for the health personnel.

\section{Acknowledgments}

Thanks is due to the University of the Philippines-National Institutes of Health for providing funds for this study. Thanks are also due to the following Medical Oncology Fellows-in-training who assisted in the interviews and specimen collection Drs. Ma Regina Valcarcel, Dennis Santos, Cherry Marquez, to Melba Baylon who assisted in the data analysis, and to Therese Marie Olivares who assisted Prof. Enriquez in the cytogenetic studies.

\section{References}

1. Hagmar L, Bonassi S, Stromberg U, et al. Cancer predictive value of cytogenetic markers used in occupational health surveillance programs. Recent Results Cancer Res. 1998; 154:177-84.

2. Hagmar L, Bonassi S, Stromberg U, et al. Cancer predictive value of cytogenetic markers used in occupational health surveillance programs: a report from an ongoing study by the European Study Group on Cytogenetic Biomarkers and Health. Mutat Res. 1998; 405(2):171-8.

3. Anderson D, Hughes JA, Cebulska-Wasilewska A, Wierzewska A, Kasper E. Biological monitoring of workers exposed to emissions from petroleum plants. Environ Health Perspect. 1996; 3:609-13.

4. Hagmar L, Stromberg U, Tinnerberg H, Mikoczy Z. The usefulness of cytogenetic biomarkers as intermediate endpoints in carcinogenesis. Int J Environ Health. 2001; 204(1):43-7.

5. Strom SS, Gu Y, Sigurdson AJ, et al. Chromosome breaks and sister chromatid exchange as predictors of second cancers in Hodgkin's disease. Leuk Lymphoma. 1998; 28(5-6):561-6.

6. Carbonell E, Demopoulos NA, Stefanou G, Psaraki K, Parry KM, Marcos R. Cytogenetic analysis in peripheral lymphocytes of cancer patients treated with cytostatic drugs: results from an EC Collaborative Study. Anticancer Drugs. 1996; 7(5):514-9.

7. Jacobson-Kram D, Albertini RJ, Branda RF, et al. Measurement of chromosomal aberrations, sister chromatid exchange, hprt mutations, and DNA adducts in peripheral lymphocytes of human populations at increased risk for cancer. Environ Health Perspect. 1993; 3:121-5.

8. Cooke J, Williams J, Morgan RJ, Cooke P, Calvert RT. Use of cytogenetic methods to determine mutagenic changes in the blood of pharmacy personnel and nurses who handle cytotoxic agents. Am J Hosp Pharm. 1991; 48 (6): 1199-205
9. Roosner P, Boffeta P, Marcello C,et al. Chromosomal aberrations in lymphocytes of healthy subjects and risk of cancer. Environ Health Perspect. 2005; 113 (5):517-520.

10. Medkova J. Cytogenetic analysis of peripheral lymphocytes in accupationally exposed health personnel. Acta Univ Palacki Olomuc Fac Med. 1990; 126:93-106.

11. Norpa H, Sorsa M, Vainio H, et al . Increased sister chromatid exchange frequencies in lymphocytes of nurses handling cytostatic drugs. Scand J Work Environ Health. 1980; 6(4):299-301.

12. Goloni-Bertollo EM, Tajara EH, Manzato AJ, Varella-Garcia M. Sister chromatid exchanges and chromosome aberrations in lymphocytes of nurses handling antineoplastic drugs. Int J Cancer. 1992; 50(3):341-4.

13. Tompa A, Magyar B, Tóth F, et al. The state of health of oncology nurses characterized by genetic and immunotoxicologic biomarkers. Magy Onkol. 2006; 60 (2):153-61.

14. Benhamou S, Pot-Deprun J, Sancho-Garnier H, Chouroulinkov I. Sister chromatid exchanges and chromosomal aberrations in lymphocytes of nurses handling cytostatic agents. In J Cancer.1998; 41(3):350-3.

15. Kasuba V, Rozgaj R, Garaj-Vrhovac V. Analysis of sister chromatid exchange and micronuclei in peripheral blood lymphocytes of nurses handling cytostatic drugs. J Appl Toxicol. 1999; 19(6):401-4.

16. Jakab MG, Major J, Tompa A. Follow-up genotoxocological monitoring of nurses handling antineoplastic drugs. J Toxicol Environ Health A. 2001; 62(5):307-18.

17. Fenech M. Chromosomal damage rate, aging, and diet. Ann NY Acad Sci. 1998; 854:23-36.

18. Bolognesi C, Abbondandolo A, Barale R, et al. Age-related increase of baseline frequencies of sister chromatid exchanges, chromosome aberrations, and micronuclei in human lymphocytes. Cancer Epidemiol Biomarkers Prev. 1997; 6(4):249-56.

19. Lazutka JR, Lekevicious R, DedonyteV, et al. Chromosomal aberrations and sister-chromatid exchanges in Lithuanian populations: effects of occupational and environmental exposures. Mutat Res. 1999; 445(2): 225-39.

20. Carere A, Andreoli C, Galati R, et al. Biomonitoring of exposure to urban air pollutants: analysis of sister chromatid exchanges and DNA lesions in peripheral lymphocytes of traffic policemen. Mutat Res. 2002; 518 (2):215-24.

21. Maki-Paakkanen J, Sorsa M, Vainio H. Sister chromatid exchanges and chromosome aberrations in rubber workers. Teratog Carcinog Mutagen. 1984; 4(2):189-200.

22. Maki-Paakkanen J, Walles S, Osterman-Golkar S, Norppa H. Singlestrand breaks, chromosome aberrations, sister chromatid exchanges and micronuclei in blood lymphocytes of workers exposed to styrene during the production of reinforced plastics. Environ Mol Mutagen. 1991; 17(1):27-31.

23. Pinto D, Ceballos JM, Garcia G, et al. Increased cytogenetic damage in outdoor painters. Mutat Res. 2000; 467(2):105-11.

24. Cardoso RS, Takahashi-Hyodo S, Peitl P Jr.,Ghilardi Neto T, Sakamoto Hojo-ET. Evaluation of chromosomal aberrations, micronuclei, and siter chromatid exchanges in hospital workers chronically exposed to ionizing radiation. Teratog Carcinog Mutagen. 2001; 21(6):431-9.

25. Donbak I, Rencuzogullari E, Topaktas M, Sahin G. A biomonitoring study on the workers from textile dyeing plants. Genetika. 2006; 42(6):757-62.

26. Joksic G, Vidakovic A, Spasojevic_Tisma V. Cytogenetic monitoring of pesticide sprayers. Environ Res. 1997; 75(2):113-8.

27. Obe G, Ristow H. Mutagenic, cancerogenic, and teratogenic effects of alcohol. Mutat Res. 1979; 65(4):229-59.

28. Sardas S, Gok S, Karakaya AE. Sister chromatid exchanges in lymphocytes of nurses handling antineoplastic drugs. Toxicol Lett. 1991; 55(3):311-5

29. Barale R, Chelotti L, Davini T, et al. Sister chromatid exchange and micronucleus frequency in human lymphocytes of 1,650 subjects in an Italian population: II. Contribution of sex, age, and lifestyle. Environ Mol Mutagen. 1998; 31(3):228-42. 